\title{
Preparation and Characterization of Cyclic Polystyrenes
}

\author{
Donghyun ChO, ${ }^{1}$ Keisuke MAsuokA, ${ }^{1}$ Kazuhiro Koguchi, ${ }^{2}$ Takeshi AsARI, ${ }^{1}$ \\ Daisuke Kawaguchi, ${ }^{1}$ Atsushi TAKano, ${ }^{1}$ and Yushu Matsushita ${ }^{1, \dagger}$ \\ ${ }^{1}$ Department of Applied Chemistry, Graduate School of Engineering, Nagoya University, \\ Furo-cho, Chikusa-ku, Nagoya 464-8603, Japan \\ ${ }^{2}$ Department of Applied Chemistry, Faculty of Engineering, Kyushu University, \\ 6-10-1 Hakozaki, Higashi-ku, Fukuoka 812-8581, Japan
}

(Received January 17, 2005; Accepted April 8, 2005; Published July 15, 2005)

\begin{abstract}
Macrocyclic polystyrenes with various molecular weight were prepared by the reaction of linear polystyrene having two end vinyl groups and potassium naphthalenide as a coupling agent. Intermolecular side reactions produced higher molecular weight polycondensates and undesirable chain termination reactions also produced linear precursors in addition to the designed molecules. In order to isolate cyclic polymers from the ring closure reaction mixtures, preparative size exclusion chromatography (SEC) method was employed. The purity of SEC-fractionated cyclic polystyrenes was rigorously examined by liquid chromatography at the critical condition (LCCC) and interaction chromatography (IC). After SEC-fractionation, we obtained large amount of highly pure cyclic polymers, though high molecular weight cyclic polymers contain very small amount of linear precursor $(<5 \%)$. The purity and isotope effect on reversed-phase liquid chromatography (RPLC) were also rigorously investigated by preparing a deuterated cyclic polystyrene. [DOI 10.1295/polymj.37.506]

KEY WORDS Cyclic Polystyrene / GPC Fractionation / LCCC / Isotope Effect /
\end{abstract}

Cyclic macromolecules are of great interest in the investigation of the influence of cyclization on their solution, melt, and solid-state properties. Various physical properties of cyclic polymers have been predicted not only by theoretical methods ${ }^{1-4}$ but also by computer simulation studies, ${ }^{5-7}$ while they have also been examined experimentally. ${ }^{8-14}$ Cyclic polymers were usually synthesized by the coupling reaction between living precursor polymers with functional groups on both ends and bifunctional linking agents. For example, polystyrenes ${ }^{9,15-19}$ and poly(2-vinylpyridine) $\mathrm{s}^{20,21}$ have been synthesized by this method. Furthermore, $\alpha, \omega$-heterobifunctional polymers have been used for the synthesis of cyclic polymers. ${ }^{22-24}$ However, side reactions produce linear precursor polymers, and intermolecular reactions simultaneously produce dimeric and higher molecular weight linear polycondensates. Therefore, it is very difficult to obtain pure cyclic polymers directly, and fractionation is necessary in order to obtain cyclic polymers with high purity. For the isolation of cyclic polymers from the ring closure reaction mixtures, two major fractionation methods have been employed: fractional precipitation method $^{9,14,16-18}$ and preparative size exclusion chromatography (SEC). ${ }^{25,26}$ Furthermore, liquid chromatography at the critical condition method (LCCC) has been successfully applied for the characterization of cyclic polymers. ${ }^{27-30}$ Throughout these experimental studies, however, the direct evidence of cyclic structure was not shown, in addition to the fact that the purity of the cyclic molecules has not been determined quantitatively in most of the works. Only for the low molecular weight cyclic polymers, the detailed cyclic structures were directly confirmed by NMR analysis of linking points, ${ }^{22,23}$ pyrolysis-gas chromatography/mass spectrometry (Py-GC/MS), ${ }^{31}$ and matrix-assisted laser desorption/ionization timeof-flight (MALDI-TOF) mass spectrometry. ${ }^{29}$ Recently, Takano et al. have reported on preparation and characterization of cyclic polystyrenes with short poly(2-tert-butyl-butadiene) sequence and proposed a novel method, ozonolysis-GPC analysis, ${ }^{32}$ to prove the purity of cyclic molecule.

In this study, we prepared cyclic polystyrenes (PS) by self-coupling in between vinyl groups on both ends of a linear precursor. By this method, cyclic PSs with molar masses of up to $600 \mathrm{~kg} / \mathrm{mol}$ can be prepared. The cyclic PSs were fractionated by preparative size exclusion chromatography (SEC) method and higher molecular weight linear polycondensates were completely removed by SEC-fractionation. SEC method, however, cannot remove completely the linear precursors from the corresponding cyclic polymers because the SEC retention time associated with hydrodynamic volume of a cyclic polymer and its linear precursor is not sufficiently different to provide complete resolution of the elution peaks. Therefore, the SEC-fractionated cyclic polymers are contaminated by linear pre-

${ }^{\dagger}$ To whom correspondence should be addressed (Tel: +81-52-789-4604, Fax: +81-52-789-3210, E-mail: yushu@apchem.nagoya-u.ac.jp). 
cursors to some extent. In order to confirm the purity of cyclic polymers, we employed two liquid chromatography methods; LCCC and interaction chromatography (IC) in this report. Generally, IC refers to the chromatography method utilizing the enthalpic interaction between the polymer chains and stationary phase while SEC separation is due to the entropic exclusion mechanism. Usually, both size exclusion and interaction mechanism are operative in the chromatographic fractionation of polymers when a porous stationary phase is employed. ${ }^{33}$

LCCC utilizes the compensation of size exclusion and interaction effect of polymer chains with porous packing materials in chromatographic separation. At the critical condition of a linear polymer species, the retention of linear polymers becomes independent of molecular weight ${ }^{34}$ while the retention of ring polymers depends on molecular weight and differs from that of linear polymers.

A deuterated-cyclic PS was also prepared and the difference in chromatographic retention behavior between hydrogenated and deuterated-cyclic PSs was rigorously investigated focusing on isotope effect in reversed-phase liquid chromatography (RPLC).

\section{EXPERIMENTAL}

\section{Materials}

The initiator, potassium naphthalenide, was synthesized by the reaction between naphthalene and potassium in THF at room temperature in vacuo. Concentration of the initiator was determined by titration with standard $\mathrm{HCl}$ solution. Tetrahydrofuran (THF), styrene, styrene- $d_{8}$ (Aldrich Chemcal Co.), and 1,1diphenylethylene (DPE) were purified using the same methods as reported previously. ${ }^{35}$ 1-[3-(3-chloropropyl)phenyl]-1-phenylethylene (I) as described below was synthesized by the $\mathrm{Li}_{2} \mathrm{CuCl}_{4}$-mediated coupling reaction of a Grignard reagent of 1-(3-bromophenyl)-1-phenylethylene with 1-bromo-3-chloropropane. The detailed synthetic procedure of I was followed by the same manners as reported by Hayashi et al. ${ }^{36}$ and the purification of I was in the same ways as reported previously. ${ }^{31,35,37}$<smiles>C=C(c1ccccc1)c1cccc(CCCl)c1</smiles>

\section{I}

\section{Preparation of Cyclic Polystyrenes}

The preparation manner of telechelic polystyrenes with DPE type vinyl groups on both ends is shown in Scheme 1. All the operations were carried out in

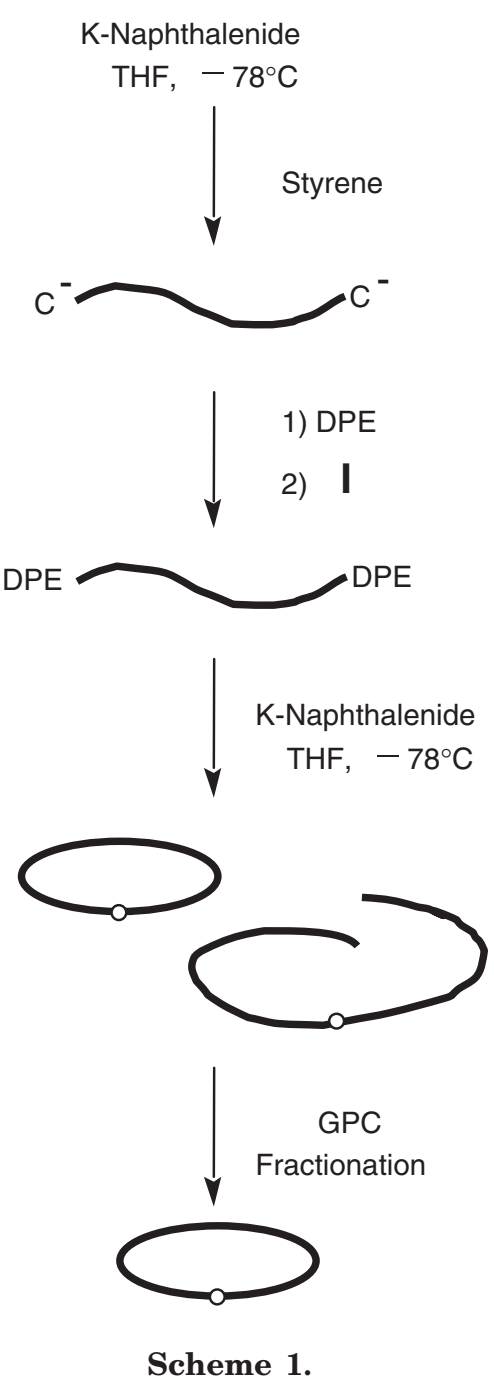

sealed glass apparatuses with breakseals under a pressure of $1 \times 10^{-3} \mathrm{~Pa}$ or lower. In order to prepare a linear telechelic polystyrene, firstly styrene was anionically polymerized with potassium naphthalenide in THF at $-78^{\circ} \mathrm{C}$, secondly DPE was added to the solution of living polymer for end-capping, and thirdly I was added for termination at room temperature. The polymers obtained were purified by precipitation in an excess amount of methanol three times to remove the residual DPE and I. After being freezedried, the telechelic polymers were transferred into vacuum glass apparatuses and then diluted with purified THF for subsequent cyclization reactions. ${ }^{35,37}$ Excess amount of potassium naphthalenide, used as the linking agent, was introduced into THF solutions $(0.05-0.2(\mathrm{w} / \mathrm{v}) \%)$ of the telechelic polymers at room temperature, and stirred for $1 \mathrm{~d}$. The stoichiometry of the molar amount of end $\mathrm{C}=\mathrm{C}$ groups on linear telechelic polymers and that of the lithium naphthalenide is $[\mathrm{Li}] /[\mathrm{C}=\mathrm{C}] \geq 5$. And the final concentrations of the coupling reaction is listed in Table I. After being quenched with dried methanol, the obtained polymer was precipitated into an excess amount of methanol. 
Table I. The final concentrations of the coupling reaction for each cyclic PSs

\begin{tabular}{cc}
\hline Sample code & $\begin{array}{c}\text { Concentration } \\
(\mathrm{g} / \mathrm{mL})\end{array}$ \\
\hline C-1 & 0.0020 \\
$\mathrm{C}-2$ & 0.0020 \\
$\mathrm{C}-3$ & 0.0020 \\
$\mathrm{C}-4$ & 0.0020 \\
$\mathrm{C}-5$ & 0.0012 \\
$\mathrm{C}_{\mathrm{d}}-5$ & 0.0009 \\
$\mathrm{C}-6$ & 0.0007 \\
\hline
\end{tabular}

\section{SEC Fractionation}

SEC fractionation was carried out at room temperature using a preparative SEC system consisting of a system controller, SC-8020, a UV detector, UV-8020, an automatic sample injector, AS-8071 and a fraction collector, FC-8010 of Tosoh. Co. equipped with a set of preparative SEC columns (two G4000HHR and one G5000HHR, $300 \times 20$ mm I.D., Tosoh. Co.). All runs for SEC-fractionation were made with THF as an eluent, where the concentrations of the polymer solutions were $c a .0 .3-0.7 \mathrm{w} / \mathrm{v} \%$. Only for the highest molecular weight sample (C-6), a precipitational fractionation in cyclohexane was carried out before SEC fractionation.

\section{Molecular Characterization}

Weight-average molecular weight, $M_{\mathrm{w}}$, was measured by light scattering in THF at $35^{\circ} \mathrm{C}$ with a multi-angle laser light scattering apparatus, MALLS Dawn-EOS of Wyatt Technology, Co. Molecular weight distribution, $M_{\mathrm{w}} / M_{\mathrm{n}}$, was determined by SEC with a set of three G 4000 HHR columns $(300 \times 7.8$ mm I.D., Tosoh. Co.). ${ }^{1} \mathrm{H}$ NMR spectra were measured with a Varian Unity Inova $500 \mathrm{MHz}$ NMR spectrometer to analyze the end-groups of telechelic polymers and connecting points of cyclic polymers. Chemical shift was referred to chloroform in chloroform- $d$. The yields of fractionated cyclic PSs after SEC fractionation and molecular characteristics are summarized in Table II.

\section{HPLC Measurements}

The IC experiments were carried out on a typical isocratic HPLC system equipped with a $\mathrm{C} 18$ bonded silica gel column (Hypersil, $100 \AA$ pore, $150 \times 4.6$ $\mathrm{mm}, 3 \mu \mathrm{m}$ particle size). The mobile phase was a mixture of $\mathrm{CH}_{2} \mathrm{Cl}_{2}$ and $\mathrm{CH}_{3} \mathrm{CN}$ (Kishida Chemical, HPLC grade, 57/43 in volume), and the flow rate was 0.5 $\mathrm{mL} / \mathrm{min}$. Each cyclic sample was dissolved in the mobile phase at the concentration of $1.0 \mathrm{mg} / \mathrm{mL}$, and injected through a Rheodyne 7125 injector equipped with a $100 \mu \mathrm{L}$ sample loop. The column temperature was adjusted by circulating a fluid through a
Table II. Molecular Characteristics of Cyclic PSs Used

\begin{tabular}{ccc}
\hline Sample code & $\begin{array}{c}M_{\mathrm{w}} \times 10^{-3 \mathrm{a}} \\
\left(M_{\mathrm{w}} / M_{\mathrm{n}}\right)^{\mathrm{b}}\end{array}$ & $\begin{array}{l}\text { Yield } \\
(\%)^{\mathrm{c}}\end{array}$ \\
\hline C-1 & $4.8(1.02)$ & 70 \\
$\mathrm{C}-2$ & $8.3(1.02)$ & 60 \\
$\mathrm{C}-3$ & $22.6(1.03)$ & 56 \\
$\mathrm{C}-4$ & $41.7(1.02)$ & 40 \\
$\mathrm{C}-5$ & $112(1.02)$ & 21 \\
$\mathrm{C}_{\mathrm{d}}-5$ & $117(1.02)$ & 20 \\
$\mathrm{C}-6$ & $573 \quad(1.03)$ & 1.8 \\
\hline
\end{tabular}

adetermined by multi angle laser light scattering bestimated by SEC ${ }^{\mathrm{c}}$ after SEC fractionation.

column jacket from a bath/circulator (HAAKE, P2, Germany). The chromatograms of cyclic PS were recorded with a UV absorption detector, UV-8020 of Tosoh. Co. operating at a wavelength of $254 \mathrm{~nm}$. For LCCC experiment, the same apparatus was employed and the column temperature was kept constant at $36.5^{\circ} \mathrm{C}$.

\section{RESULTS AND DISCUSSION}

Figure 1 displays the SEC chromatograms of three linear precursors; i.e., L2, L4, and L5, and the corresponding raw products after cyclization reactions having different molecular weights. From the SEC chromatograms of three raw products, we found that the higher molecular weight polycondensates were formed besides cyclic molecules in the course of cyclization reaction of telechelic polymers. To remove these undesirable side-products, the SEC fractionation was carried out by preparative SEC. The fractionation

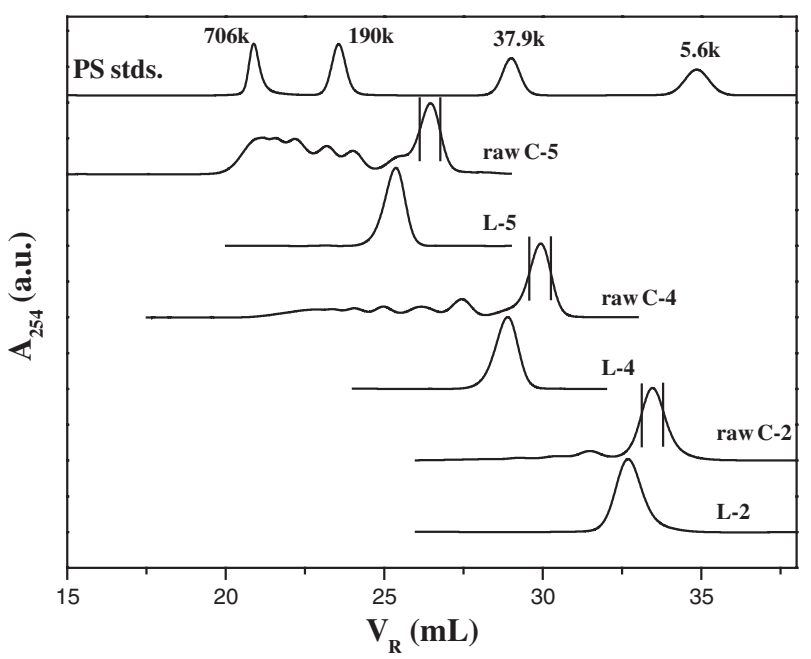

Figure 1. SEC chromatograms of three linear precursors, i.e., L2, L4 and L5 and the corresponding raw products just after cyclization reactions. The double vertical bars are indicative of SEC fractionation areas for each raw products. 


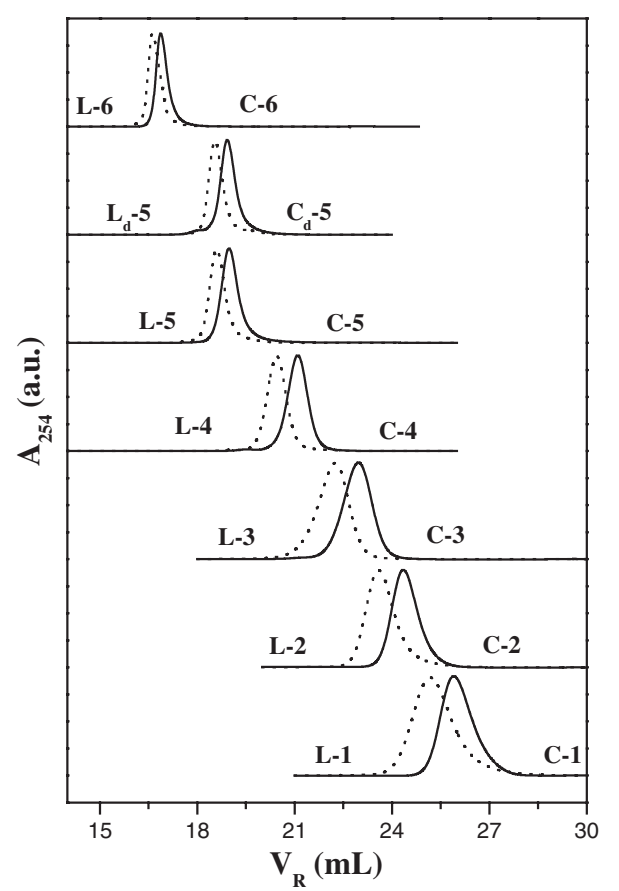

Figure 2. SEC chromatograms of fractionated cyclic PSs (solid lines) and the corresponding linear precursors (dotted lines). All the linear PS precursors are eluted at smaller retention volume than cyclic PSs, though the elution peaks are partially overlapping.

areas were also shown in Figure 1, where the double vertical bars indicate the boundaries of each fraction.

In Figure 2 are displayed the SEC chromatograms of the fractionated six hydrogenated-cyclic PSs and one deuterated-cyclic PS $\left(\mathrm{C}_{\mathrm{d}}-5\right)$ expressed by solid lines together with those of their linear precursors by dotted lines. From SEC chromatograms of all cyclic PSs, it is evident that the higher molecular weight polycondensates in raw-products were completely removed by SEC-fractionation, and that every cyclic PSs are eluted at longer retention volume than their linear precursors since the ring closure causes the reduction in the hydrodynamic volume of the polymer chains. The difference in the hydrodynamic volume between linear and cyclic PS is, however, not sufficiently large to provide their complete resolution in SEC. Accordingly, although the SEC chromatograms of cyclic PSs do not show any trace of their linear precursors, it is still probable for the fractionated polymer to contain very small amount of linear precursors. In short, it is difficult to quantify the linear contaminants in the fractionated-cyclic PSs by SEC measurements only.

In Figure 3, LCCC chromatograms of cyclic PSs with five different molecular weights and one deuterated-cyclic PS at the critical condition for linear hydrogenated PSs are displayed. The small peaks appearing around $4 \mathrm{~min}$ are for the linear hydrogenated PSs and the injected solvent. The linear PSs are

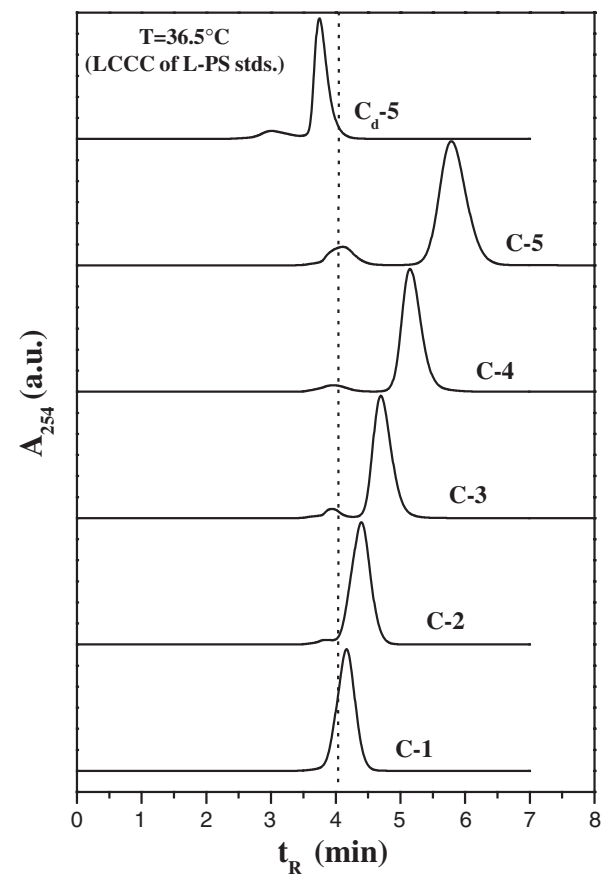

Figure 3. LCCC chromatograms of the fractionated cyclic PSs at the chromatographic critical condition for linear PS. The small peaks appearing at near $t_{\mathrm{R}}=4 \mathrm{~min}$ are the duplication of the peaks for the linear precursors and that for the injected solvent. Column: Hypersil C18, $100 \AA$ A $150 \times 4.6 \mathrm{~mm}$, Eluent: $\mathrm{CH}_{2} \mathrm{Cl}_{2}$ / $\mathrm{CH}_{3} \mathrm{CN}(57 / 43, \mathrm{v} / \mathrm{v})$ at a flow rate of $0.5 \mathrm{~mL} / \mathrm{min}$.

eluted at almost the same retention time independent of molecular weight, which is the typical elution behavior of the linear polymer chains at the chromatographic critical condition. From the LCCC chromatograms of cyclic PSs, it is clear that all the cyclic PSs are successfully purified by SEC-fractionation. At this elution conditions, we found from Figure 3 that the retention time of cyclic PSs increases with increasing the molecular weight and finally, the cyclic PSs are completely separated from their linear precursors. At the same time, it has been found that the elution behavior of deuterated-cyclic polymer is completely different from that of the other hydrogenated polymers. That is, deuterated-cyclic PS is eluted even before the injected solvent, which means that deuterated-cyclic PS eluted in SEC region at the same separation condition, whereas all the hydrogenated-cyclic PSs are eluted in interaction chromatography (IC) region. For this different behavior of deuterated-cyclic PS, we will discuss more in detail later.

Although LCCC method shows very high resolution in the separation between linear and cyclic PSs, it is difficult to quantify the small amount of linear contaminant because the elution peaks of linear PSs were mostly overlapped with injected solvent peaks. Therefore, to quantify the amount of linear PSs accurately, we employed the reversed-phase interaction liquid chromatography (RPLC) method. Figure 4 


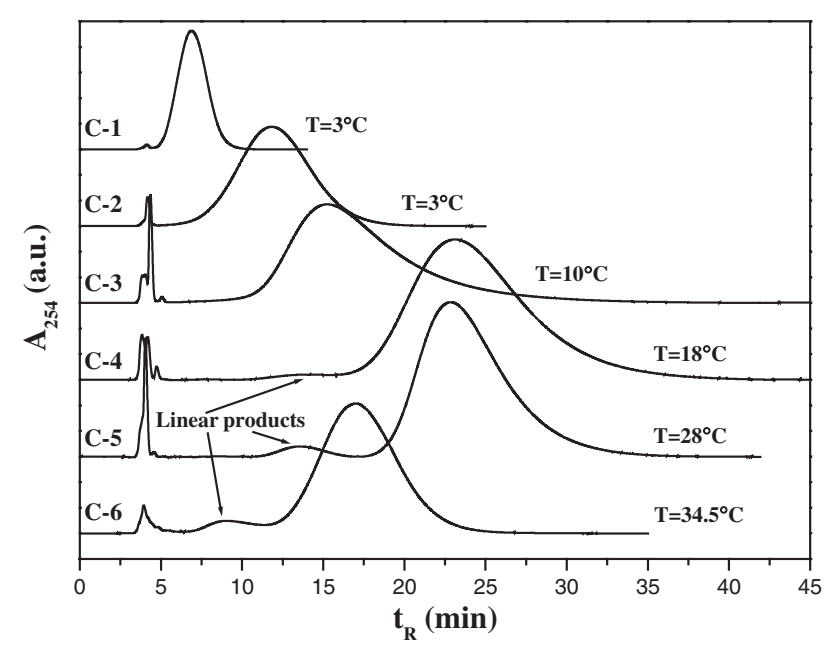

Figure 4. RPLC chromatograms of six different SEC-fractionated cyclic PSs in Table I. The elution peaks of C1, C2, and C-3 are all single showing high purity of the cyclic PSs, while the small peaks at lower $t_{\mathrm{R}}$ sides of C-4, C-5, and C-6 are for linear precursors contained in each fractionated-PS.

shows the RPLC chromatograms of six hydrogenatedcyclic PSs with different molecular weight. The column temperature was adjusted so that all the cyclic PSs elute in a reasonable IC elution time. From the RPLC chromatograms, we found that the three lower molecular weight cyclic PSs, C-1, C-2, C-3, show broad single peaks only, which means that these three fractionated cyclic PSs are not contaminated with linear precursors at all. For the three C-4, C-5 and C-6 cyclic PSs, to the contrary, small peaks can be seen at the left of the major peaks. These small peaks correspond to linear side-products. From the RPLC chromatograms of C-4, C-5 and C- 6 , the amount of linear contaminant is estimated to be below $5 \%$, this value is very small considering relatively high molecular weights of cyclic polymers.

On the other hand, in Figure 5, RPLC chromatograms of hydrogenated-cyclic PS (C-5) (A) and a deuterated-cyclic PS $\left(\mathrm{C}_{\mathrm{d}}-5\right)(\mathrm{B})$ are compared together with that of the linear deuterated-PS (B) represented by a dotted line at the constant temperature of $28^{\circ} \mathrm{C}$. From the SEC chromatograms of $\mathrm{C}-5$ and $\mathrm{C}_{\mathrm{d}}-5$ in Figure 2, although the molecular weights of C-5 and $\mathrm{C}_{\mathrm{d}}-5$ are almost identical, the retention time of $\mathrm{C}_{\mathrm{d}}-5$ is completely different from that of C-5. Normally a deuterated polymer is eluted at much shorter elution time than the normal counterpart if reversed-phase silica gel is used. This unusual retention behavior of a deuterated-cyclic PS obviously comes from the isotope effect. In general, hydrogenated and deuterated molecules can be regarded as almost identical with respect to their chemical behavior as well as most physical ones, however, isotope labeling affects markedly the retention in IC, ${ }^{38,39}$ especially, in reversed-

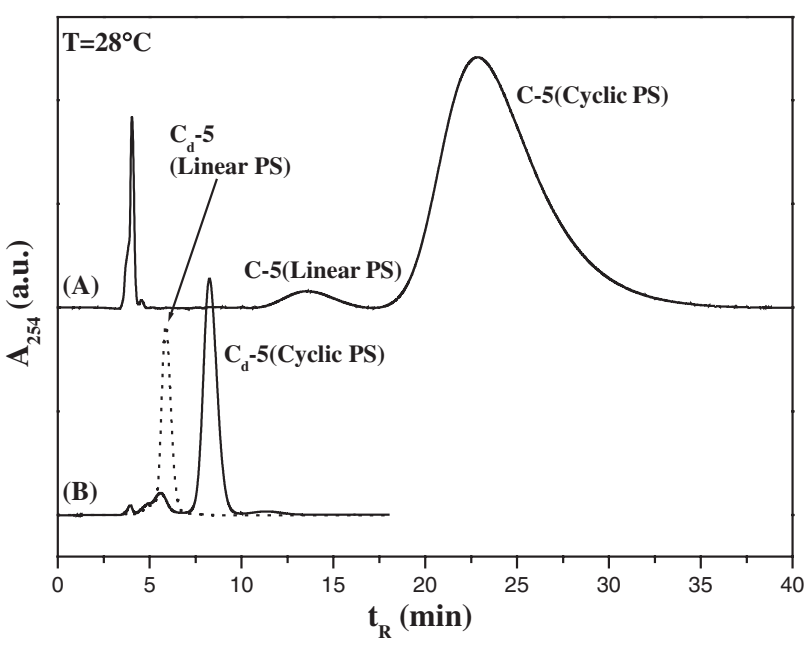

Figure 5. RPLC chromatograms of a hydrogenated-cyclic PS (C-5, A) and a deuterated-cyclic PS, $\mathrm{C}_{\mathrm{d}}-5$ expressed by a solid line at $\mathrm{B}$ and the corresponding linear deuterated-PS drawn by a dotted line at B at constant temperature. Deuterated-cyclic PS is eluted at lower $t_{\mathrm{R}}$ than hydrogenated-cyclic PS for this RPLC system. Column: Hypersil C18, $100 \AA, 150 \times 4.6 \mathrm{~mm}$, Eluent: $\mathrm{CH}_{2} \mathrm{Cl}_{2}$ / $\mathrm{CH}_{3} \mathrm{CN}(57 / 43, \mathrm{v} / \mathrm{v})$ at a flow rate of $0.5 \mathrm{~mL} / \mathrm{min}$.

phase stationary phase. Perny et al. ${ }^{40}$ reported this kind of isotope labeling effect between hydrogenated and deuterated-linear polybutadiene samples in RPLC. For this isotope effect, we have also found that a deuterated cyclic PS could be separated completely from the corresponding a hydrogenated cyclic PS by RPLC due to the difference in chromatographic behavior in this paper.

In conclusion, using a new coupling agent, cyclic PSs can be prepared with molar masses up to 600 $\mathrm{kg} / \mathrm{mol}$. From the SEC-fractionation, we completely removed the higher molecular weight polycondensates and obtained certain amount of highly pure single chain cyclic PSs. And the purity of SEC-fractionated cyclic PSs has been accurately determined by LCCC and RPLC analysis. These highly pure cyclic polymers can be applied for the systematic investigation of various physical properties of cyclic polymers in comparison with linear counterparts in the near future.

Acknowledgment. This work was done under the support of one of the 21st century COE programs entitled "The Creation of Nature-Guided Materials Processing" in the School of Engineering Nagoya University, Nagoya, Japan.

\section{REFERENCES}

1. H. A. Kramers, J. Chem. Phys., 14, 415 (1946).

2. B. H. Zimm and W. H. Stockmayer, J. Chem. Phys., 17, 1301 (1949). 
3. E. F. Casassa, J. Polym. Sci., A, 3, 605 (1965).

4. W. Burchard and M. Schmidt, Polymer, 21, 745 (1980).

5. J. Naghizadeh and A. Sotobayashi, J. Chem. Phys., 60, 3104 (1974).

6. W. Baumgartner, J. Chem. Phys., 76, 4275 (1982).

7. J. M. Garcia Bernal, J. J. Tirado, M. M. Freire, and J. Garcia de la Torre, Macromolecules, 23, 3357 (1990).

8. J. S. Higgins, K. Dodgson, and A. Semlyen, Polymer, 20, 553 (1979).

9. B. Vollmert and J. X. Huang, Makromol. Chem., Rapid Commun., 2, 467 (1981).

10. J. Roovers, Macromolecules, 18, 1359 (1985).

11. G. Hadziioannou, P. M. Cotts, G. ten Brinke, C. C. Han, P. Lutz, C. Strazielle, P. Remmp, and A. J. Kovacs, Macromolecules, 20, 493 (1987).

12. P. J. Mills, J. W. Mayer, E. J. Kramer, G. Hadziioannou, P. Lutz, C. Strazielle, P. Remmp, and A. J. Kovacs, Macromolecules, 20, 513 (1987).

13. J. Roovers, Macromolecules, 21, 1517 (1988).

14. J. Roovers and P. M. Toporowski, J. Polym. Sci., B, 26, 1251 (1988).

15. G. Hild, A. Kohler, and P. Remmp, Eur. Polym. J., 16, 525 (1980).

16. D. Geiser and H. Hoeker, Macromolecules, 13, 653 (1980).

17. B. Vollmert and J. Huang, Makromol. Chem., Rapid Commun., 1, 333 (1980).

18. J. Roovers and P. M. Toporowski, Macromolecules, 16, 843 (1983).

19. K. Ishizu and H. Kanno, Polymer, 37, 1487 (1996).

20. W. Toreki, T. E. Hogen-Esch, and G. B. Butler, Polym. Prepr., Am. Chem. Soc. Div. Polym. Chem., 28, 343 (1987).

21. W. Toreki, T. E. Hogen-Esch, and G. B. Butler, Polym. Prepr., Am. Chem. Soc. Div. Polym. Chem., 29, 17 (1988).

22. L. Rique-Lurbet, M. Schappacher, and A. Deffieux, Macromolecules, 27, 6318 (1994).

23. M. Kubo, T. Hayashi, H. Kobayashi, K. Tsuboi, and T. Itoh, Macromolecules, 30, 2805 (1997).
24. B. Lepoittevin, X. Perrot, M. Masure, and P. Hemery, Macromolecules, 34, 425 (2001).

25. K. Dodgson, D. Sympson, and J. A. Semlyen, Polymer, 19, 1285 (1978).

26. A. C. Dagger and J. A. Semlyen, Polymer, 40, 3243 (1999).

27. H. C. Lee, H. Lee, W. Lee, T. Chang, and J. Roovers, Macromolecules, 33, 8119 (2000).

28. B. Lepoittevin, M. A. Dourges, M. Masure, P. Hempery, K. Baran, and H. Cramail, Macromolecules, 33, 8218 (2000).

29. D. Cho, S. Park, K. Kwon, T. Chang, and J. Roovers, Macromolecules, 34, 7570 (2001).

30. S. Singla, T. Zhao, and H. W. Beckham, Macromolecules, 36, 6945 (2003).

31. H. Ohtani, H. Kotsuji, H. Momose, Y. Matsushita, and S. Tsuge, Macromolecules, 32, 6541 (1999).

32. A. Takano, A. Nonaka, O. Kadoi, K, Hirahara, S. Kawahara, Y. Isono, N. Torikai, and Y. Matsushita, J. Polym. Sci., B, 40, 1582 (2002).

33. G. Glockner, "Gradient HPLC of Copolymers and Chromatographic Cross-Fractionation," Springer-Verlag, Berlin, 1992.

34. B. G. Belenky, E. S. Gankina, M. B. Tennikov, and L. Z. Vilenchik, Dokl. Akad. Nauk, 231, 1147 (1976).

35. A. Takano, K. Kondo, M. Ueno, K. Ito, S. Kawahara, Y. Isono, J. Suzuki, and Y. Matsushita, Polym. J., 33, 5539 (2001).

36. M. Hayashi, S. Nakahama, and A. Hirao, Macromolecules, 32, 1325 (1999).

37. A. Takano, O. Kadoi, K. Hirahara, S. Kawahara, Y. Isono, J. Suzuki, and Y. Matsushida, Macromolecules, 36, 3045 (2003).

38. P. D. Klein, Adv. Chromatogr., 3, 3 (1996).

39. N. Tanaka and E. R. Thornton, J. Am. Chem. Soc., 98, 1617 (1976).

40. S. Perny, J. Allgaier, D. Cho, W. Lee, and T. Chang, Macromolecules, 34, 5408 (2001). 